\title{
Android Application to get Answer through Voice
}

\author{
Mr. Prof. P. D. Hasalkar, Ms. Masaratjahan I. Kudle, Mr. Prasad N. Chaudhari, \\ Mr. Deepak B. Mali, Ms. Mohammadi M. Kamplee \\ Walchand Institute of Technology, Solapur
}

\begin{abstract}
The mobile phone users are increasing day by day and everyone wants to develop new things which are improved version of existing one. The user might want the faster technology which can give the answer by giving input in the form of voice and getting output in the form of voice. This project deals with the easy to use and time saving feature and providing the easy search facility.

In this technology, there will be voice-to-text and text-to-voice interaction between the user and the server. Here first voice is converted into text and this text is searched in the database residing in application itself and extracts its answer, then this answer will be converted into voice. If answer is not found in database then it will sent over Google to extract its answer and gets converted into voice. Finally the client will be getting the answer in the form of a voice for the searched result.
\end{abstract}

Keywords: Speech-to-text conversion (STT), Text-to-speech conversion (TTS)

\section{INTRODUCTION}

People love their mobile phones because they can actually stay in touch wherever they are. That means not just for talking, but e-mailing, texting, and so on. We are constructing with the growth of mobile phone technology. As per the users are increase day by day, facilities are also increasing. Let's start with simple regular handsets which were used just for making phone calls, mobiles have changed our lives and have become part of it. Now a day's mobile phones are not use for making calls but they have innumerable uses and can be used as a Camera, Music player, Tablet PC, T.V. ,Web browser etc. And with the new technologies, new software and operating systems are required. In recent years, smart phones have placed an increasing emphasis on bringing speech technologies into mainstream usage. This focus has led to products such as Speech server, which is used to implement speech-enabled telephony systems. Now let's limit our focus towards applications of android mobile phones. Finding answer on Google is the most widely used application in the world, with 2.4 billion active users. But instead of searching the answer by typing, we just give input in the form of voice by just speaking the question of which we required answer and also getting its answer in form of voice itself minimizing the work.

\section{AIM AND OBJECTIVE}

The main goal of our project is to develop android application for getting Answer by giving input as well as getting output in the form of voice.

\section{Objective includes}

A. Speech to text conversion

This will include the conversion of speech into text by matching appropriate peach and frequencies, which is done by servers holding large database regarding possible different peach and frequencies of a person's voice. This server is already built by Google

\section{B. Searching mechanism}

After converting speech into text the searching mechanism is performed which include searching of that answer in the database residing in application itself and extracting its meaning.

\section{Connecting to Google via internet}

If the desired text does not match with any answer in database then it will search that answer over Google through internet.

\section{Text to speech conversion}

If the answer is found then it will be converted into voice as an output of our application.

\section{EXISTING SYSTEM}

Whenever we want to get answer of any question we need to perform searching task in the huge database thus wasting lot of time. This process involves considerable wastage of time as it is not always possible to type question or not to read its answer. The process requires human interaction at each state and takes a lot of time. Briefly we can summaries the drawback as:

\section{A. Manually typing of question}

In the existing system, whenever you want to find the answer from mobile phone you need to type whole question manually.

\section{B. Time Consuming}

As each and every time we need to type whole question, search accordingly and also need to read its answer thus wasting lot of time.

\section{PROBLEM STATEMENT}

User gives input in the form of voice, this voice is converted into text and this text is searched in the databse residing in application itself and extracts its answer, then 
this answer will be converted into voice. If answer is not found in mobile-database then it will be sent over Google to extract its answer and gets converted into voice. Finally the client will be getting a answer in the form of a voice for the searched result.

\section{IMPLEMENTATION}

Here we are using eclipse for writing android source code for designing interface and creating various processes included in application. We begin with the conversion of the voice into text, this is performed by matching the person's voice peach and frequency then finding the appropriate question, this task is performed by Google servers having huge database regarding different peach and frequencies then the resultant text is searched in the database through searching technique and if the question is found its corresponding answer is extracted and given as input to the another process where this text is converted into voice, the text to voice conversion takes place internally and we are getting output as voice saying the answer of the question which we have spoken as input. If the answer is not found in the database then it is sent over the Google to search the answer, the first paragraph after that word is extracted and stored in the temporary storage space and also given for further processing where it actually gets converted into voice as final output. For storing the answer and creating temporary storage space the database is required. For this purpose we are using SQlite database which is very simple and suitable mini database system. Separate space as temporary storage has to be made to store the question and its answer which is not found in the database but searched over the Google, this question along with its answer is stored in the temporary storage for quick accessing of questions answer instead of again searching it over the Google[1]. The flow diagram of the whole system shows in the Fig.1

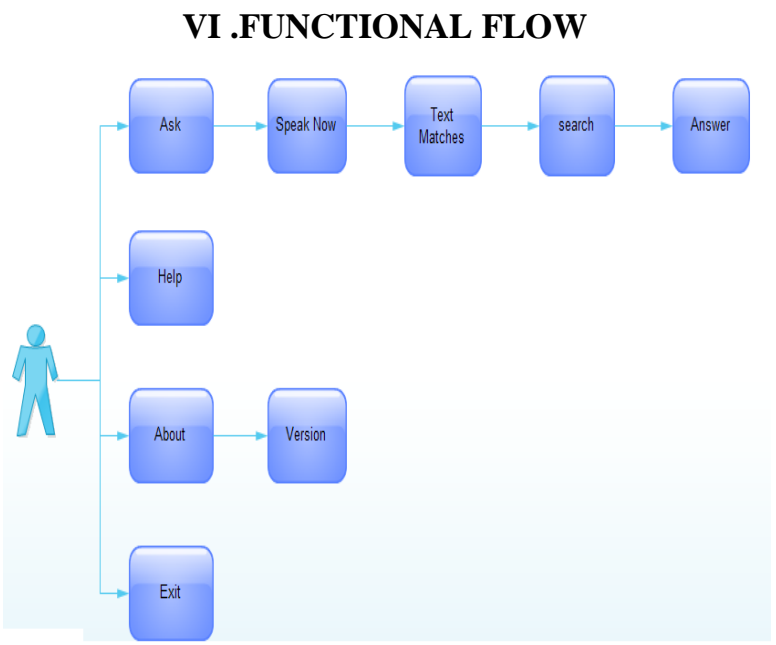

Fig.1.Flow of proposed application

\section{UNITS}

A. speech-to-text conversion

The main requirement of every speech to text conversion system is a database which will compare peach with frequencies. If we develop the system which will convert the speech into text globally that is for any user it is very difficult job because the frequency of giving input of any user is different as that of other user. If the system is global hence we are creating it for the mobile user means our job is very much difficult there are millions and billions mobile users and sound frequency and peach comparison is again difficult. Hence we need to use database which is already provided by "Google". If we try to create the database manually it will create time and space problem. To create such a huge database lot of time will west and this project is for mobile users whose internal memory of mobile is generally low and the database for this is system is very much huge. Instead of creating a database for this system we need to use existing database which is available at the web server. The speech input is collected in the container and the send it to the peach and frequency comparison there cognition take place. To get correct text which is spoken we need training on input file and the output is collected in the text file.

\section{B. SEARCHING MECHANISM}

The system includes three searching mechanisms are as listed below:

$i$. Searching in database- After converting the speech into the text the searching mechanism search the answer in the database residing in mobile itself and extract the answer of the question. If the answer of question is not found in the database then it searches in the temporary storage.

ii. Searching in temporary storage- In this it searches the answer of question in the temporary storage and extracts the answer of the question. This searching mechanism stores the answer for a particular slot of time. If the answer is not found in the temporary storage then it will be searched over Google.

iii. Searching over Google- Google search the answer of the question and extract the answer of it. This extracted answer is stored in temporary Storage for some period of time.

\section{TEXT-TO-SPEECH}

Text to speech synthesis is converting the text to the synthetic speech that is as close to real speech as possible according to the pronunciation norms of special language. Such systems are called text to speech (TTS) systems. Input element of TTS system is a text, output element is synthetic speech. where the actual conversion is done by storing some rules regarding text analyzing like saving pronunciation of each alphabets with some exceptions, taking pause whenever space is been encountered while actual converting text into speech and so on.

VIII. DETAILS OF SOFTWARE AND HARDWARE. A. Software Requirements Operating System:-Android operating system. $B$. Hardware Requirements Mobile phone with specification.

- $\quad$ Android operating system (version 2.3). $650 \mathrm{MHz}$ processor. 512 MB RAM. 


\section{CONCLUSION}

This paper will demonstrate the plan and implementation of building an application which will give answer in the form of voice having user friendly interface and useful in day to day life for many users for finding the answer of the question. This will also overcome on various disadvantages found in existing system thus making it more efficient and reliable.

\section{REFERENCES}

1. www.google.com

2. www.wikipedia.com

3. "Android Application Development All in one for Dummies" by Barry Burd

4. "Mobile Apps Development" by Anubhav Pradhan, Anil V Deshpande

5. "Embedded Android-Porting, Extending, and Customizing" by Karim Yaghmour

6. (O'Reilly Media)

7. Android Developer Resources: http://developer.android.com

8. Android Developer Tools Essentials by Mike Wolfson (O'Reilly Media). 